Homology, Homotopy and Applications, vol.10(3), 2008, pp.15-25

\title{
W. STEPHEN WILSON'S CONTRIBUTIONS TO HOMOTOPY THEORY
}

\author{
MARTIN BENDERSKY
}

(communicated by Donald M. Davis)

\begin{abstract}
This paper is a survey of Steve's work on $B P$ and periodic cohomology theories. It was presented as a talk given in March 2007 at a conference celebrating Steve's 60th birthday.
\end{abstract}

\section{Cohomology of $\Omega$-spectra and Hopf rings}

Steve Wilson's thesis, directed in 1972 by Frank Peterson, started unstable BP theory as we know it. Part 1 of his thesis appeared as [13], where he shows that the spaces in the $\Omega$-spectrum for $B P$ are as nice as one may expect.

Theorem 1.1. $\mathbb{Z}_{(p)}$-cohomology of $\underline{B P}_{k}$, the $k$-th space in the $\Omega$ spectrum for $B P$, is torsion free. As a Hopf algebra it is a bi-free algebra; i.e., the homology and cohomology are free algebras.

The original proof in his thesis goes back to Brown and Peterson's definition of $B P$ as the inverse limit of spaces inductively defined by a series of fibrations. Steve uses the Eilenberg-Moore spectral sequence of these Brown-Peterson fibrations to prove the theorem.

This is not the way we now understand the homology of the spaces in the $B P \Omega$ spectrum, or for that matter the generalized homology of the spaces in the $\Omega$-spectrum of any multiplicative homology theory. The relevant technology is the language of Hopf rings developed by Ravenel and Wilson in [10].

The idea here is to exploit the structure one has after packaging all of the spaces in the $B P \Omega$-spectrum together. Milgram used this structure when he computed the homology of $B G$. The homology of the individual spaces are obviously $H$-spaces. But now we can mix the different factors together using the ring structure. This gives us two products, the $*$ (loop space)-product and a $\circ$ product

$$
\circ: B P_{*}\left(\underline{B P}_{n}\right) \otimes B P_{*}\left(\underline{B P}_{k}\right) \rightarrow B P_{*}\left(\underline{B P}_{n+k}\right) .
$$

A Hopf ring is a graded Hopf algebra with an additional product satisfying a list of relations which makes it a ring object in the category of coalgebras. The collection $\left\{B P_{*}\left(\underline{B P}_{n}\right)\right\}$ is an example.

Received August 7, 2007, revised August 15, 2007; published on December 5, 2008.

2000 Mathematics Subject Classification: 55N22, 55Q51, 55S25.

Key words and phrases: cobordism, $v_{n}$-periodic homotopy.

This article is available at http://intlpress.com/HHA/v10/n3/a2

Copyright (C) 2008, International Press. Permission to copy for private use granted. 
Now add a geometric relation coming from the natural map

$$
\mathbb{C} P^{\infty} \times \mathbb{C} P^{\infty} \rightarrow \mathbb{C} P^{\infty} \text {. }
$$

Stably this induces the formal group law. Unstably this induces relations between the $\circ$ and * products. Their main theorem is that $B P_{*}(\underline{B P} *)$ is completely determined by the Hopf ring structure and this relation. As a corollary this reproves the results in Steve's thesis. They also give a basis for the indecomposables and the primitives. In other words they give us what we need to start applying unstable $B P$ to problems in homotopy theory.

Since Hopf rings play such an important role in Steve's work, I would like to say a few words about the structure in the case of complex cobordism. Milnor gave a very nice description of the stable image of the dual of the unstable Steenrod algebra. For $p>2$ let's write a class in the polynomial algebra part of the dual Steenrod algebra as

$$
\xi_{1}^{a_{1}} \xi_{2}^{a_{2}} \cdots \xi_{k}^{a_{k}} \otimes x_{n}
$$

(Here $\xi_{i}$ is a generator in dimension $2\left(p^{i}-1\right)$ that comes from $\mathbb{C} P^{\infty}$, and $x_{n}$ is an $n$-dimensional class.) The excess condition in the Steenrod algebra dualizes to

$$
2 \Sigma a_{i} \leqslant n
$$

That is to say, classes that satisfy this condition lift to the homology of the $n$-th Eilenberg-MacLane space. The same thing happens for $B P$. For all primes, there are classes $b_{i} \in B P_{2\left(p^{i}-1\right)}(B P)$ such that $b_{1}^{a_{1}} b_{2}^{a_{2}} \cdots b_{k}^{a_{k}} \otimes x_{n}$, with the same excess condition on the $a_{i}$ 's, generate the stable image of $Q B P_{*}\left(\underline{B P}_{n}\right)$. Since the suspension is an injection on the indecomposables, this tells us what the indecomposables look like back in the Hopf ring. Of course the situation is more complicated for $B P$. First of all, the suspension is a rational isomorphism. If you play around with the right action, you start to see how torsion appears in the cokernel of the map

$$
Q B P_{*}\left(\underline{B P}_{n}\right) \rightarrow B P_{*} B P,
$$

and you see how classes in the cokernel vanish after some suspensions. For example, it is easy to check that the class $b_{1}^{2}$ is an element of order $p$ in the cokernel of the suspension from $B P_{*}\left(\underline{B P}_{2}\right)$ which is obviously in the image of $B P_{*}\left(\underline{B P}_{4}\right)$. Steve and Doug, using the bar spectral sequence and some fancy algebra, exploit the right action to come up with a basis for the indecomposables and the primitives (the RavenelWilson basis. There are at least three others).

With this tool in hand, Steve, often with Doug, went on to compute the generalized cohomology of the spaces for numerous $\Omega$-spectra. One particularly important example is the Morava $K$-theory of the $\bmod p^{j}$ Eilenberg-MacLane spaces that appeared in a paper with Doug Ravenel, $[\mathbf{1 1}]$. Let $\mathbf{K}_{*}$ be the product of the $\bmod p^{j}$ EilenbergMacLane space. They prove

Theorem 1.2. The Hopf ring for $K(n)_{*}\left(\mathbf{K}_{*}\right)$ is the free Hopf ring over $K(n)_{*}\left(\mathbf{K}_{0}\right)$ generated by $K(n)_{*}\left(\mathbf{K}_{1}\right)$.

The fact that the $K(n)$-homology of all of the Eilenberg-MacLane spaces is generated as a Hopf ring by the first space in the $\Omega$-spectrum is one of the beauties of 
Hopf ring technology. In particular, a simple Gysin sequence calculation shows that $K(n)_{*}\left(\mathbf{K}_{1}\right)$ is concentrated in even degree. From this it follows that the Morava $K$ theory of all of the Eilenberg-MacLane spaces is concentrated in even degrees. Other examples where the Hopf ring is generated by few classes is the Hopf ring for $B P$ (generated by $B P_{*}\left(\underline{B P}_{2}\right)$, a suspension operator and the lifts of the right action) and the $\bmod p$ homology of the mod $p$ Eilenberg-MacLane spaces (a well-known calculation) generated as a Hopf ring by $H_{*}\left(\mathbf{K}_{i} ; \mathbb{Z}_{p}\right), i=0,1,2$.

The Morava's "little" structure theorem allows Steve and Doug to glue together all the information they have for $K(n)_{*}\left(\mathbf{K}_{*}\right)$ to compute the annihilator ideal for the canonical element $\iota_{n} \in B P_{n}(\mathbf{K}(\mathbb{Z} /(p), n))$ ( $p$ is odd $)$.

\section{Theorem 1.3.}

$$
\operatorname{Ann}\left(\iota_{n}\right)=\left(p, v_{1}, \cdots, v_{n-1}\right) \subset B P_{*} .
$$

This has geometric consequences, the so-called Conner-Floyd conjecture. In their book on differentiable periodic maps, Conner and Floyd were interested in the bordism element $\gamma_{n}=\left(T^{n} \rightarrow B \mathbb{Z} /(p)^{n \times}\right)$. They conjectured that

$$
\operatorname{Ann}\left(\gamma_{n}\right)=\left(p, M^{2(p-1)}, \cdots, M^{2\left(p^{n-1}-1\right)}\right) \subset M S O_{*},
$$

where $M^{2\left(p^{j}-1\right)}$ are the Milnor manifolds. They were able to prove that the annihilator ideal contained this ideal. Steve and Doug's computation implies the other inclusion.

The obvious question to ask is why not calculate $A n n\left(\gamma_{n}\right)$ by simply computing $B P_{*}(B \mathbb{Z} /(p) \times \cdots \times B \mathbb{Z} /(p))$ ? This computation is very hard and was not available in 1980 .

$B P_{*}$ was not known for many spaces in the early 80 's. This situation had to be rectified in order to justify the developing technology. $B P_{*}(B \mathbb{Z} /(p) \times \cdots \times B \mathbb{Z} /(p))$ was an important test case. Conner, Floyd and Landweber and Stong computed the $n=2,3$ cases. The breakthrough calculation was the work of Steve and Dave Johnson published in [5].

Here they compute the associated graded group of a $B P_{*}$-module filtration of $B P_{*}(B \mathbb{Z} /(p) \times \cdots \times B \mathbb{Z} /(p))$. The filtration was unraveled in a paper by Steve, Dave Johnson and Dung Yan $[6]$.

Interestingly, their work does not provide a new proof of the Conner-Floyd conjecture In fact one uses the validity of the Conner-Floyd conjecture as input to compute $B P_{*}(B \mathbb{Z} /(p) \times \cdots \times B \mathbb{Z} /(p))$.

Unstable $B P$ cohomology operations are much more subtle than the unstable Steenrod algebra. First of all, one has to give a precise definition of the ring of unstable operations. Once that is done, one needs the notion of the category of unstable module over the ring of unstable operations. This has been worked out in all the detail one would ever want for general cohomology theories in Steve's paper, [1] with Mike Boardman and Dave Johnson.

I actually like Steve's approach in his BP Introduction and Sampler [15]. If one just wants to just understand $B P$ operations, I prefer reading 40 or so pages to 150 ! (not factorial).

The point I want to emphasize is that there are additive unstable operations that do not come from stable operations. Briefly, for $n>0 B P_{\mathbb{Q}}^{k-n} B P \simeq P B P_{\mathbb{Q}}^{k}\left(\underline{B P}_{n}\right)$. (A bit of a warning here. $B P_{\mathbb{Q}}^{*} B P$ is the cohomology theory, $B P_{\mathbb{Q}}^{*}$ of $B P$. This is not 
the same as $B P^{*} B P \otimes \mathbb{Q}$.) If we restrict to $k$-dimensional complexes, the coker in the sequence

$$
B P^{*-n} B P \rightarrow P B P^{*}(\underline{B P})_{n} \rightarrow \text { coker }
$$

is torsion. This is the whole story for $k$-dimensional complexes. One may think of an unstable operation as a sum of stable operations with some powers of $p$ in the denominators. To see which powers give an actual unstable cohomology operation, one "simply" dualizes the homology calculation in the Hopf rings paper. However if we wish to understand unstable cohomology operations for arbitrarily large $k$, the situation is a bit more bizarre. Steve defines a truly unstable operation to be an additive unstable operation (i.e. an element of $P B P^{*}(\underline{B P})$ ) which is not a stable operation. In cohomology we can take infinite sums. So it is reasonable to think of a truly unstable operation as an unstable operation which is an infinite sum of stable operations with arbitrarily large powers of $p$ in the denominators. Steve shows that this does indeed describe all truly unstable operations and proves the disturbing fact:

"no multiple of a truly unstable operation is ever stable; although ratio-

nally, unstable and stable operations are the same."

If you crave an example of a truly unstable operation, there is the example of the generalized Adams operations defined using the bracket series

$$
\psi^{k}(x)=\frac{[k](x)}{k} .
$$

\section{Spectra related to $B P$ and the Wilson $Y_{k}$ 's}

Here is a (redundant) list of some of the $B P$-related theories:

- $B P\langle n\rangle=B P_{*} /\left(v_{i} \mid i>n\right)$.

- $E(n)_{*}=v_{n}^{-1} B P\langle n\rangle_{*}$.

- $P(k, n)_{*}=B P\langle n\rangle /\left(p, v_{1}, \cdots, v_{k-1}\right), 0 \leqslant k \leqslant n \leqslant \infty(P(k)=P(k, \infty))$.

- $E(k, n)_{*}=v_{n}^{-1} P(k, n)_{*}$.

This list includes Johnson-Wilson theory, connective and periodic Morava $K$-theory and $B P(=P(0))$. (Actually for technical reasons $P(0)$ is often the $p$-adic completion of $B P$. I will ignore this bit of subtlety here.) Steve and his coauthors use the obvious interlocking exact sequences between these theories for varying $k$ and $n$ to compute the $B P$ homology and cohomology of interesting spaces.

$B P\langle n\rangle$ is important in Part II of Steve's thesis, [14]. In this paper, he finds the indecomposable factors in a product decomposition of the spaces in the $\Omega$-spectrum for $B P$. The factors, $\left\{Y_{k}\right\}$, are not only the building blocks for the $\underline{B P}_{n}$; they are the building blocks for any $H$-space with torsion-free homotopy and torsion-free $\mathbb{Z}_{(p)^{-}}$ homology. I would like to mention two applications of this result. The fact that $H$-spaces with torsion-free homotopy and homology, e.g., $S U$ and $\underline{B P}_{n}$, split up into the same spaces implies that the unstable Adams-Novikov spectral sequence for such spaces collapses to the 0-line. This is the only way I know how to prove this. It is the starting point for my work with Don Davis on the $v_{1}$-periodic homotopy groups of $S U(n)$. 
The spaces $\left\{Y_{k}\right\}$ do not quite form an $\Omega$-spectrum. Indeed,

$$
\Omega Y_{2 \frac{p^{r}-1}{p-1}+1} \approx Y_{2 \frac{p^{r}-1}{p-1}} \times Y_{2 p \frac{p^{r}-1}{p-1}},
$$

but they are close enough for Alex Zabrodsky to construct Adams resolutions using $Y_{k}$. There are applications of these resolutions in Alex's beautiful book, Hopf Spaces $([\mathbf{2 0}])$.

A few words about where the $Y_{k}$ 's come from. Steve uses Baas and Sullivan's theory of manifolds with singularities to construct the $Y_{k}$ 's. First he constructs the homology theories $B P\langle n\rangle$. Then he shows that for $2 \frac{p^{n}-1}{p-1}<k \leqslant 2 \frac{p^{n+1}-1}{p-1}$ the spaces in the $\Omega$-spectrum, $\underline{B P\langle n\rangle} \underline{k}_{k}$, are particularly nice. $Y_{k}$ is defined to be $\underline{B P\langle n\rangle} \underline{k}_{k}$ for $k$ in this range.

The Hopf algebra $H^{*}\left(\frac{B P\langle n\rangle}{2 k}\right)$ is bipolynomial for $0<k<2 \frac{p^{n+1}-1}{p-1}$. Bipolynomial Hopf algebras over $\mathbb{Z}_{(p)}$ are classified in a neat paper with Doug Ravenel, [9]. In particular, the Hopf algebra structure for $H^{*}\left(Y_{k}\right)$ is completely determined.

As $n$ increases, the cohomology theories $B P\langle n\rangle$ interpolate from $\bmod p$ homology to $B P$. In [4] Dave Johnson and Steve use this and the results of part II of Steve's thesis to give seven criteria equivalent to the homological dimension over $M U_{*}$ for $M U_{*}(X)$ being $\leqslant n+1$, generalizing results of Conner-Smith and Johnson-Smith. Specifically, Johnson and Smith prove that the homological dimension over $M U_{*}$ of $M U_{*}(X)$ is $\leqslant 1$ if and only if multiplication by $t$ in $k_{*}(X)$ is monic $\left(k_{*}(X)\right.$ is connective $K$-theory). Dave and Steve replace $k_{*}$ with the theories $B P\langle n\rangle_{*}$ Steve introduced in his thesis. Multiplication by $t$ naturally gets replaced with multiplication with $v_{n}$. In Section 3 of this paper they prove one step by localizing $B P\langle n\rangle(X)_{*}$. We now denote the resulting Johnson-Wilson theory by $E(n) \quad\left(E(n)_{*}=v_{n}^{-1} B P\langle n\rangle_{*}\right)$. Using the methods of the proof, they also show in a remark that $E(n)_{*}(X)=B P_{*}(X) \otimes_{B P_{*}}$ $E(n)_{*}$. This formula may look familiar. This says that $E(n)$ is an example of a Landweber exact homology theory. In fact Peter wrote his celebrated paper to reprove results in this paper.

\section{3. $B P$ homology and cohomology of interesting spaces}

A test for an exotic homology theory is whether one can compute it for interesting spaces. Steve has made as much progress as anyone has making such computations for the homology theories derived from $B P$.

I already mentioned his work with Johnson and Yan computing $B P_{*}$ of the elementary $p$-groups. The result is that the hard work computing $B P_{*}$ of the classifying space of an elementary abelian $p$-group of rank $n$ involves the first $n$ coefficients. The rest get carried along for the ride.

Theorem $3.1([6])$. If $G$ is an elementary abelian p-group of rank $n$, then there is an isomorphism of abelian groups

$$
B P_{*}(B G)=B P\langle n\rangle_{*}(B G) \otimes_{\mathbb{Z}_{(p)}} \mathbb{Z}_{(p)}\left[v_{n+1}, \cdots\right] .
$$

Tony Bahri, Don Davis, Peter Gilkey and I proved this for the rank-one case and stated the higher-rank case as a conjecture. 
Steve makes the case that computing $B P^{*}(X)$ is often easier than computing $B P_{*}(X)$. Peter Landweber's computation of $B P^{*}(B G)$ for an abelian $G$ is much easier. There is a family of spaces for which Steve and his coauthors tell us a lot about their BP cohomology. These are spaces which have their Morava $K$-theory concentrated in even degree. Now except for $K(\mathbb{Z}, 1)$, the Morava $K$-theory of EilenbergMacLane spaces has this property. Furthermore since

$$
K(n)^{*} X \simeq \operatorname{hom}_{K(n)_{*}}\left(K(n)_{*} X, K(n)_{*}\right),
$$

the same is true for $K(n)$-cohomology. This has implications for the BP-cohomology of the loops on an $H$-space with finite Postnikov tower.

These spaces are studied in a paper that Steve wrote with Mike Hopkins and Doug Ravenel. They prove in [3]:

Theorem 3.2. For $X$ an $H$-space with finitely many nonzero homotopy groups,

$$
K(n)_{*}(\Omega X) \simeq \bigotimes_{0 \leqslant m \leqslant n+1} K(n)_{*}\left(K\left(\pi_{m}(\Omega X), m\right)\right) .
$$

The condition $n+1 \geqslant m$ is not a surprise since $K(n)_{*}$ thinks a large EilenbergMacLane space is a point.

So, in particular if $X$ has a finite Postnikov tower, then $K(n)_{*}\left(\Omega^{2} X\right)$ has the same $K(n)$-homology as a product of Eilenberg-MacLane spaces. These were computed in the Conner-Floyd paper mentioned above.

There is another family of spaces whose Morava $K$-theory is the same as for Eilenberg-MacLane spaces. These appear as the spaces in the $\Omega$-spectrum of strongly $K(n)_{*}$-acyclic spectra.

Theorem 3.3. Let $X=\left\{\underline{X}_{i}\right\}$ be a connective $\Omega$-spectrum with $K(n)_{*}\left(\underline{X}_{m}\right) \simeq K(n)_{*}$ for some $m$. Then for all $k$

$$
K(n)_{*}\left(\underline{X}_{k}\right) \simeq \bigotimes_{n+1 \geqslant i \geqslant 0} K(n)_{*}\left(K\left(\pi_{i}\left(\underline{X}_{k}\right), i\right)\right)
$$

Notice all it took was $K(n)_{*}$ of one space in the $\Omega$-spectrum to look like a point to force all of them to look like Eilenberg-MacLane spaces. This forces the spectrum, $\mathbf{X}$, to be $K(n)$-acyclic (called strongly acyclic). It is not the case that a $K(n)$-acyclic spectrum must have a $K(n)$-acyclic space in its $\Omega$-spectrum.

The results of Hopkins, Ravenel and Wilson are input to [12] written with Doug and Nobuaki Yagita. This paper establishes once and for all that it is easier to compute $B P$ cohomology than homology, at least for spaces with even Morava $K$-theory, the very spaces studied in $[\mathbf{3}]$. There are a lot of nice theorems in this paper. I will mention a few. The standing hypothesis is that $X$ is a space with even Morava $K$-theory.

Theorem 3.4. For $E=E(k, n)$ or $P(k), E^{*}(X)$ is concentrated in even dimensions and is flat and finitely presented as an $E^{*}$-module.

As a consequence, this says that there is no $v_{k}$-torsion in $P(k)^{*}(X)$.

So, for example, this applies to the loops on spaces with finite Postnikov tower. We cannot say the same for the spaces that appear in the $\Omega$-spectrum of a strongly acyclic spectrum. Namely, being strongly acyclic for $K(n)$ does not imply even acyclic for 
$K(n+1)$. However the classifying spaces of most finite groups, $Q S^{2 n}, B O(n), M O(n)$ and many other spaces have even $K(n)$ cohomology.

Theorem 3.5. There is a Künneth isomorphism

$$
E^{*}(X \times Y) \simeq E^{*}(X) \widehat{\otimes}_{E^{*}} E^{*}(Y) .
$$

In particular, if we know $P(0)^{*}$ (which is essentially $B P$ ) for $K\left(\mathbb{Z}_{(p)}\right)$ and for $K\left(\mathbb{Z} /\left(p^{i}\right)\right)$, we know it for the Eilenberg-MacLane spaces of any finitely generated abelian group. The computation of the $P(0)$-cohomology of the factors is Theorem 1.14 in their paper. Interestingly, the answer involves the spaces in the $\Omega$-spectrum for $B P\langle q\rangle$, which goes back to part II of Steve's thesis. So we now know $B P^{*}$ for the double loops of all spaces with finite Postnikov tower.

As mentioned, $B O(n)$ has even $K(n)$ cohomology. I think Steve was hoping that there might be an exotic characteristic class in $B P^{*}(B O(n))$, just what one would want to get new obstructions to geometric dimensions. Unfortunately, while $B P^{*}(B O(n))$ is hard to compute, it is easy to describe. There are the Conner-Floyd classes, $C_{k}$, of the complexification of the canonical bundle, and there is the relation which says that $C_{k}=C_{k}^{*},\left(C_{k}^{*}\right.$ is the Conner-Floyd class of the complex conjugate bundle). Steve's theorem says that there are no other classes nor other relations. This computation appears in [17].

There are real versions of Johnson-Wilson spectra, $E R(n)$, constructed by $\mathrm{Hu}$ and Kriz. For example, $E R(2)$ is a 48-periodic theory which Steve used in a recent paper with Nitu Kitchloo to obtain nonimmersions of real projective spaces, [7].

The crux of the paper is to use a Bockstein spectral sequence to compute the ring $E R(2)^{*}\left(R P^{2 n}\right)$. Before I give an example of a new nonimmersion, I would like to give a bit of history. I think Steve has been interested in the nonimmersion problem from the beginning of his career. In his book, A BP Introduction and Sampler, ([15]), he used unstable $B P$ to obtain a nondesuspension for $R P_{16}^{26}$. This particular nondesuspension result does not give a nonimmersion. Don Davis and I were able to extend Steve's idea to formulate a conjecture

$$
R P^{2(m+\alpha(m)-1)} \nsubseteq \mathbb{R}^{2(m-\alpha(m))},
$$

which we were able to prove for small $\alpha(m)$. In [16] Steve was able to extend the result to more values of $m$ after showing that the conjecture followed if a particular element was of order two, and Don proved it for all $m$ in his Annals paper using a $B P\langle 2\rangle$ Euler class. I would like to emphasize the point that the conjecture jumps out at you when you hit the nonimmersion problem with unstable $B P$, but the computations are too difficult in that context. With the conjecture provided by the unstable theory, it became provable using stable methods. Steve's work with Nitu improves on Don's theorem by two dimensions in some special cases. At this time, with all of the work of Don Davis, Mark Mahowald and others, new low-dimensional nonimmersions are hard to obtain. For example, the first new immersion claimed in a difficult paper of Bruner, Davis, and Mahowald ([2]) using tmf shows that

$$
R P^{1536} \nsubseteq \mathbb{R}^{3036} \text {. }
$$

Compare this with Steve and Nitu's lowest new nonimmersion:

$$
R P^{48} \nsubseteq \mathbb{R}^{84}
$$


(I should mention that late in 2006 Davis and his coauthors realized that they had overlooked some low-dimensional cases in [2] that overlap with Steve and Nitu's results.)

It has taken 20 years to have a new nonimmersion for a low-dimensional projective space.

\section{The periodic phenomena paper}

One of the most important papers in Steve's oeuvre is his paper with Haynes Miller and Doug Ravenel, [8]. Here the ideas of Jack Morava on periodicity in the stable homotopy groups of spheres were exploited to give insight into the structure of the Adams-Novikov spectral sequence that still resonates throughout homotopy.

A bit more history: Adams, Smith and Toda started the program of constructing periodic families of elements, $\alpha_{t}, \beta_{t}, \gamma_{t}$ in $\pi_{*}\left(S^{0}\right)$. The $\gamma$ family was the most delicate to detect. In fact $\gamma_{1}$ was a point of contention in the early 70's. Thomas and Zahler, using $B P$, claimed to have shown that $\gamma_{1} \neq 0$, while the Toda school claimed it was 0 . There was even an article in the New York Times with the headline "A Contradiction in Mathematics." The outcome was that Thomas and Zahler were correct. They went on to detect infinitely many, but not all, of the gamma family. One of the main achievements of the periodic phenomena paper was to detect all of the gammas. They did this by completely computing the 2-line of the Adams-Novikov spectral sequence. Using $v_{1}, v_{2}$ and $v_{3}$, they construct classes, $\beta_{s p^{n} / i, j+1} \in E_{2}^{2,2\left(p^{2}-1\right) s p^{n}-2(p-1) j}$. The class is $p^{i+1}$ - and $v_{1}^{j}$-torsion. They determine the constraints on $i, j, s$ and $n$ so the resulting subcollection of $\beta_{s p^{n} / i, j+1}$ 's generates a direct sum of cyclic groups.

One of the applications of this computation is obtained by mapping to the classical Adams spectral sequence. The result is that most of the elements in the Adams 2-line do not survive. Steve thinks this is the result that got people's attention.

It was Adams who made the case that homology was more tractable than cohomology. Steve's papers do show that computing $B P^{*}$ may be easier than $B P_{*}$ for important spaces. But when it comes to setting up the Adams spectral sequence, I think this work makes it clear that Adams was right. Zahler made some significant progress using the cohomology approach to the Novikov spectral sequence. But his methods were stymied by the complex computations and gave no hint of the periodic behavior of the spectral sequence.

\section{Steve's work on Mathematics Education}

As hard as it is to believe, most people actually think our work as teachers is more important than computing the homotopy groups of spheres. Steve is a mathematician who wears both hats. While working on his paper with Nitu, he was commuting to Washington D.C. to advise the Department of Education on mathematics education issues (perhaps he should mention the commuter rail, MARC, in the acknowledgments).

I particularly like [19] where Steve uses Johns Hopkins students to demonstrate that those who became dependent on calculators did worse in their college mathematics classes. (I would like to add a minor observation: I've noticed that students 
who do arithmetic with a calculator often think the sequence

$1.0,1.1,1.2,1.10,1.11$

is increasing.)

There was, as expected, a reaction from the mathematics education community to which Steve responded in [18]. I can't improve on Steve's words, so here they are:

Now I get into touchy territory. I am well aware of how the so-called standard algorithms for arithmetic have fallen on hard times. Educators frequently dismiss them as obsolete and unnecessary. On the contrary, from the point of view of the teacher who gets your students next, I cannot emphasize enough how important these basic algorithms (or their equivalent) are. There are many reasons for this and properly dealing with them would require a paper in itself. Briefly,

1. the standard algorithms are the only collection of beautiful, serious, mathematical theorems you can teach to a child in K-6. They are amazingly powerful. They take the ad hoc out of arithmetic. They extend observed patterns and give the operations structure. These theorems solve the age-old problem of how to do basic computations without having to use different strategies for different numbers. The mystery is gone.

2. Students will be confronted with new algorithms constantly as they progress in their study of mathematics. Ignoring the most basic and most important of all algorithms is not good preparation.

3. In high school and college mathematics, these very same algorithms will be slightly modified and generalized and used in different settings. This happens many times over and a mastery of the original algorithms makes this process an incremental one rather than an overwhelming one.

Given the thrilling description of the standard algorithms I have just relayed, I found Tunis's final comment disheartening: "How can we give them the idea that mathematics is more than the rote learning of algorithms?" Again, I would turn this around and ask "How could anyone get the idea that mathematics is just the rote learning of algorithms?" If the standard algorithms are taught with understanding, enthusiasm and admiration, then a student should find them exciting and appreciate the awesome power they give.

Steve is still thinking about these issues. He recently included a link on Don's discussion group proving that our students are getting worse. Steve is the only person studying mathematics education who has the data! He traces the deterioration to the NCTM standards.

"Nineteen eighty-nine is, in mathematics education, indelibly tied to the National Council of Teachers of Mathematics' publication, Curriculum and Evaluation Standards for School Mathematics (1989), which downplayed pencil and paper computations and strongly suggested that calculators play an important role in K-12 mathematics education. My 2006 students would have been about two years old at the time of this very influential 
publication, and it could easily have affected the mathematical education many of them received. Certainly, one possibility is that mathematics preparation is down across the country, thus limiting the pool of well prepared college applicants."

The foundational work of Steve and his coauthors has described the beautiful stable and unstable structure of $B P$ and related periodic theories. His work has had enormous application. It is a pleasure to thank Steve for his contribution to homotopy theory and wish him a happy birthday.

\section{References}

[1] J. M. Boardman, D. C. Johnson, and W. S. Wilson, Unstable operations in generalized cohomology, Handbook of Algebraic Topology, 687-828, North-Holland, Amsterdam (1995).

[2] R. Bruner, D. M. Davis, and M. Mahowald, Nonimmersions of real projective spaces implied by tmf, Contemporary Mathematics 293 (2002) 45-68.

[3] M. J. Hopkins, D. C. Ravenel, and W. S. Wilson, Morava Hopf algebras and spaces $K(n)$-equivalent to finite Postnikov systems, Fields Institute Communications 19 (1998), 137-163.

[4] D. C. Johnson and W. S. Wilson, Projective dimension and Brown-Peterson homology, Topology 12 (1973), 327-353.

[5] D. C. Johnson and W. S. Wilson, The Brown-Peterson homology of elementary p-groups, American Journal of Mathematics 107 (1985), 427-453.

[6] D. C. Johnson, W. S. Wilson and D. Y. Yan, Brown-Peterson homology of elementary p-groups II, Topology and its Applications 59 (1994), 117-136.

[7] N. Kitchloo and W. S. Wilson, The second Real Johnson-Wilson theory and nonimmersions of $R P^{n}$, preprint.

[8] H. R. Miller, D. C. Ravenel, and W. S. Wilson, Periodic phenomena in the Adams-Novikov spectral sequence, Annals of Mathematics 106 (1997), 469516.

[9] D. C. Ravenel and W. S. Wilson, Bipolynomial Hopf algebras, Journal of Pure and Applied Algebra 4 (1974), 41-45.

[10] D. C. Ravenel and W. S. Wilson, The Hopf ring for complex cobordism, Journal of Pure and Applied Alg. 9 (1977), 241-280.

[11] D. C. Ravenel and W. S. Wilson, The Morava $K$-theories of Eilenberg-MacLane spaces and the Conner-Floyd conjecture, American Journal of Mathematics 102 (1980), 691-748.

[12] D. C. Ravenel, W. S. Wilson, and N. Yagita, Brown-Peterson cohomology from Morava K-theory, K-Theory 15 (1998), 147-199.

[13] W. S. Wilson, The $\Omega$-spectrum for Brown-Peterson cohomology, Part I, Commentarii Mathematici Helvetici 48 (1973), 45-55. 
[14] W. S. Wilson, The $\Omega$-spectrum for Brown-Peterson cohomology Part II, American Journal of Mathematics 97 (1975), 101-123.

[15] W. S. Wilson, Brown-Peterson Homology: an introducton and sampler, CBMS Regional Conf. Series 48 (1982).

[16] W. S. Wilson, Brown-Peterson metastability and the Bendersky-Davis conjecture, Publ. Res. Inst. Math. Sci. 20 (1984), 1037-1051.

[17] W. S. Wilson, The complex cobordism of $B \mathrm{O}_{n}$, Journal of the London Mathematical Society 29 (1984), 352-366.

[18] W. S. Wilson, Short response to Tunis's letter to the editor on technology in college, Educational Studies in Mathematics 58 (2005), 415-420.

[19] W. S. Wilson and D. Q. Naiman, K-12 calculator usage and college grades, Educational Studies in Mathematics 56 (2004), 119-122.

[20] A. Zabrodsky, Hopf Spaces, North-Holland Mathematics Studies 22 (1976).

Martin Bendersky mbenders@hunter.cuny.edu

Department of Mathematics and Statistics, Hunter College, 695 Park Avenue, New York, NY 10065, USA 